\title{
Kinetic Studies of Glutamate Dehydrogenase
}

\author{
THE REDUCTIVE AMINATION OF 2-OXOGLUTARATE
}

\author{
By P. C. ENGEL AND K. DALZIEL \\ Department of Biochemistry, University of Oxford, Oxford OX1 3QU, U.K.
}

(Received 6 March 1970)

\begin{abstract}
1. Kinetic studies of the reductive amination of 2-oxoglutarate catalysed by glutamate dehydrogenase with NADH and NADPH as coenzyme were made at pH 7.0 and pH 8.0. The concentrations of both substrates and coenzymes were simultaneously varied over wide ranges. Lineweaver-Burk plots with respect to each substrate and coenzyme were linear, except that with high concentrations of 2-oxoglutarate or coenzyme inhibition occurred. There was no evidence of the negative homotropic interactions between the enzyme subunits that were revealed in previous kinetic studies of the reverse reaction. 2. The initial-rate results are shown to be inconsistent with any of the six possible compulsory-order mechanisms for this three-substrate reaction, and it is concluded that a random-order mechanism is the most likely one. On the basis of this mechanism, the dissociation constants of all the binary, ternary and quaternary complexes of the enzyme and substrates are calculated from initial-rate parameters. 3. The results are discussed in relation to those of earlier workers who concluded that the mechanism is of the compulsoryorder type.
\end{abstract}

Several plausible mechanisms for enzyme reactions involving three substrates can be distinguished by initial-rate measurements, provided that the concentrations of all three substrates are varied simultaneously, so that all the parameters in the initial-rate equation can be evaluated (Dalziel, 1969). In previous kinetic studies of the reductive amination of 2-oxoglutarate catalysed by glutamate dehydrogenase (EC 1.4.1.3) each substrate in turn was kept at a high constant concentration while the concentrations of the other two substrates were varied (Frieden, 1959; Corman, Prescott \& Kaplan, 1967; Fahien \& Strmecki, 1969). It was concluded by Frieden (1959) that with the ox liver enzyme a compulsory-order mechanism is followed, the quaternary complex being formed by successive combination of NADPH, $\mathrm{NH}_{4}{ }^{+}$and 2-oxoglutarate with the enzyme. Corman et al. (1967) concluded that the same mechanism applies to the enzyme from dogfish liver. On the other hand, Fahien \& Strmecki (1969) proposed the sequence NADH, 2-oxoglutarate, $\mathrm{NH}_{4}{ }^{+}$from their studies of the ox liver enzyme in arsenate buffer, and the sequence NADPH, $\mathrm{NH}_{4}{ }^{+}, 2$ 2-oxoglutarate in tris-acetate buffer.

The results of more detailed kinetic studies, both with NADH and with NADPH as coenzyme, are reported in this paper, and are inconsistent with a compulsory-order mechanism. Earlier kinetic studies of the oxidative deamination of glutamate by NAD and NADP showed a complex pattern of 'coenzyme activation', which was attributed to negative homotropic interactions between enzyme subunits (Dalziel \& Engel, 1968; Engel \& Dalziel, 1969). The present studies of the reverse reaction show no evidence of such interactions. LineweaverBurk plots are linear over wide ranges of coenzyme and substrate concentrations, but inhibition with high concentrations of either reduced coenzyme or 2-oxoglutarate is confirmed (Olson \& Anfinsen, 1953).

\section{MATERIALS AND METHODS}

Materials. Glutamate dehydrogenase from ox liver was prepared and assayed as previously described (Engel \& Dalziel, 1969). NADPH was obtained from C. F. Boehringer und Soehne G.m.b.H., Mannheim, Germany. The sodium salt of NADH was prepared from NAD as described by Dalziel (1962) except that the intermediate isolation of the barium salt was omitted. Both reduced coenzymes were assayed by extinction measurements at $340 \mathrm{~nm}$ by using the extinction coefficient of $6.22 \times 10^{6} \mathrm{~cm}^{2} / \mathrm{mol}$ (Horecker \& Kornberg, 1948).

2-Oxoglutaric aoid obtained from Nutritional Biochemicals Corporation, Cleveland, Ohio, U.S.A., and from Koch-Light Laboratories, Colnbrook, Bucks., U.K., was recrystallized once from acetone-benzene without change of m.p. $\left(114^{\circ} \mathrm{C}\right)$. Solutions were adjusted to the required 
$\mathrm{pH}$ with $\mathrm{NaOH}$ and assayed as described by Londesborough \& Dalziel (1968).

Other chemicals were of analytical grade from British Drug Houses Ltd., Poole, Dorset, U.K. Solutions of $\left(\mathrm{NH}_{4}\right)_{2} \mathrm{HPO}_{4}$ were adjusted to the required $\mathrm{pH}$ value with $\mathrm{H}_{3} \mathrm{PO}_{4}$ and kept tightly stoppered.

Kinetic measurements. Initial-rate measurements were made at $25^{\circ} \mathrm{C}$ in $0.111 \mathrm{~m}$-sodium phosphate buffers, $\mathrm{pH} 7.0$ and 10.25 , and in $0.087 \mathrm{~m}$-sodium phosphate buffer, pH 8.0 and I0.25, containing $10 \mu \mathrm{M}$-EDTA. Most of the measurements were made with a recording fluorimeter as described by Engel \& Dalziel (1969). For rate measurements with large NADH concentrations $(>30 \mu \mathrm{M})$ a Zeiss model PMQ II spectrophotometer was used.

Evaluation of initial-rate parameters. Initial-rate results for a three-substrate reaction which yield linear Lineweaver-Burk plots with respect to each substrate with any fixed concentrations of the other two may be described in general by an equation of the form:

$$
\begin{array}{r}
\frac{e}{v_{0}}=\phi_{0}+\frac{\phi_{\mathrm{A}}}{[\mathrm{A}]}+\frac{\phi_{\mathrm{B}}}{[\mathrm{B}]}+\frac{\phi_{\mathrm{C}}}{[\mathrm{C}]}+\frac{\phi_{\mathrm{AB}}}{[\mathrm{A}][\mathrm{B}]}+\frac{\phi_{\mathrm{AC}}}{[\mathrm{A}][\mathrm{C}]}+\frac{\phi_{\mathrm{BC}}}{[\mathrm{B}][\mathrm{C}]}+ \\
\frac{\phi_{\mathrm{ABC}}}{[\mathrm{A}][\mathrm{B}][\mathrm{C}]}
\end{array}
$$

In this equation (Dalziel, 1969), $e$ is the enzyme concentra- tion, $v_{0}$ is the initial rate and [A], [B] and [C] are the sub. strate concentrations. To evaluate all eight kinetic coefficients $\phi_{0}, \phi_{A}$ etc., initial-rate measurements are made with several concentrations of each substrate in all possible combinations. From primary Lineweaver-Burk plots of $e / v_{0}$ against $1 /[\mathrm{A}]$ for example, for several fixed values of $[B]$ and $[C]$, slopes and intercepts are obtained and are plotted against the reciprocals of each of the two latter concentrations. The slopes and intercepts of these four secondary plots are then plotted against the reciprocal of the remaining concentration variable, and two estimates of each of the eight parameters in eqn. (1) are obtained as slopes and intercepts of these eight tertiary plots. The same initial-rate results can also be plotted with $1 /[B]$ or $1 /[C]$ as the primary variable, yielding altogether six estimates of each parameter and some indication of their reliability. The procedure is illustrated and described in detail by Dalziel (1969).

Ideally, the concentration ranges of the three substrates used should embrace conditions in which each term in eqn. (1) is dominant in turn. In practice, the ranges are limited on the one hand by the sensitivity of the method used for rate measurements, and on the other by the common occurrence of substrate inhibition with high concentrations.

As in earlier work (Engel \& Dalziel, 1969) a molecular
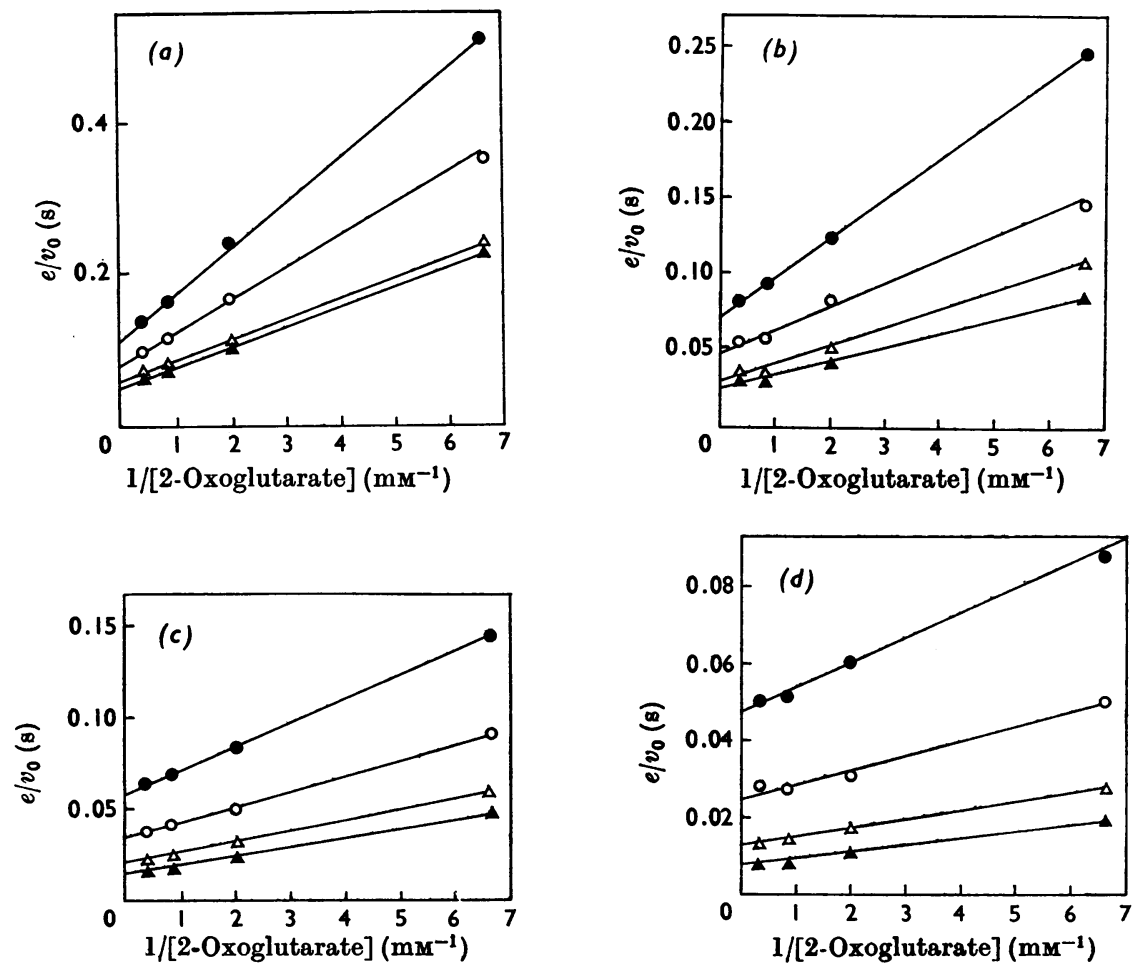

Fig. 1. Primary Lineweaver-Burk plots of initial-rate results with the concentration of 2-oxoglutarate as variable and four fixed NADH concentrations $(\mu \mathrm{M}):(a) 0.411 ;(b) 1.03 ;(c) 2.05 ;(d) 6.15$. In each graph, the $\mathrm{NH}_{4}{ }^{+}$concentrations (mM) are: $\bullet, 1.5 ; 0,3.0 ; \triangle, 7.5 ; \Delta, 18.0$. Phosphate buffer, pH 7.0 and $I 0.25$, was used. 
weight of $10^{6}$ has been assumed for the enzyme in order to express $e$ in molar units and simplify the dimensions of the kinetic parameters.

\section{RESULTS}

Kinetics with $N A D H$ at $\mathrm{pH} 7.0$. Initial-rate measurements were made with four concentrations of each substrate in the ranges 0.411-6.15 $\mu \mathrm{M}$ $\mathrm{NADH}, \quad 0.15-3.0 \mathrm{~mm}-2$-oxoglutarate and 1.5$18.0 \mathrm{~mm}-\mathrm{NH}_{4}{ }^{+}$. The enzyme concentration was $0.225 \mu \mathrm{g} / \mathrm{ml}$. Primary plots of $e / v_{0}$ against the reciprocal of the concentration of 2-oxoglutarate are shown in Fig. 1 and are linear. Secondary plots of the slopes and intercepts against the reciprocals of the concentrations of each of the other two substrates (Fig. 2) are also linear within the experimental error. Finally, the slopes and intercepts of these secondary plots are plotted against the recipro- cal of the concentration of the remaining substrate in Fig. 3. From these tertiary plots, two values for each of the eight initial-rate parameters in eqn. (1) are obtained directly as slopes and intercepts. The expressions for the slopes and intercepts of the primary and secondary plots of Figs. 1 and 2 can readily be derived from eqn. (1), with $A=2$-oxoglutarate, $\mathrm{B}=\mathrm{NH}_{4}{ }^{+}$and $\mathrm{C}=\mathrm{NADH}$, and were given by Dalziel (1969). The ways in which the kinetic coefficients are obtained from the tertiary plots are indicated in the legends to Fig. 3, and were also illustrated by Dalziel (1969).

Two alternative sets of primary plots of the initial-rate results with $\mathrm{NADH}$ and $\mathrm{NH}_{4}{ }^{+}$concentration as variable, and the appropriate secondary and tertiary plots, were also made, but are not shown, except that some of the tertiary plots are included in Fig. 3. In general, appropriate pairs of tertiary plots were in satisfactory agreement, and
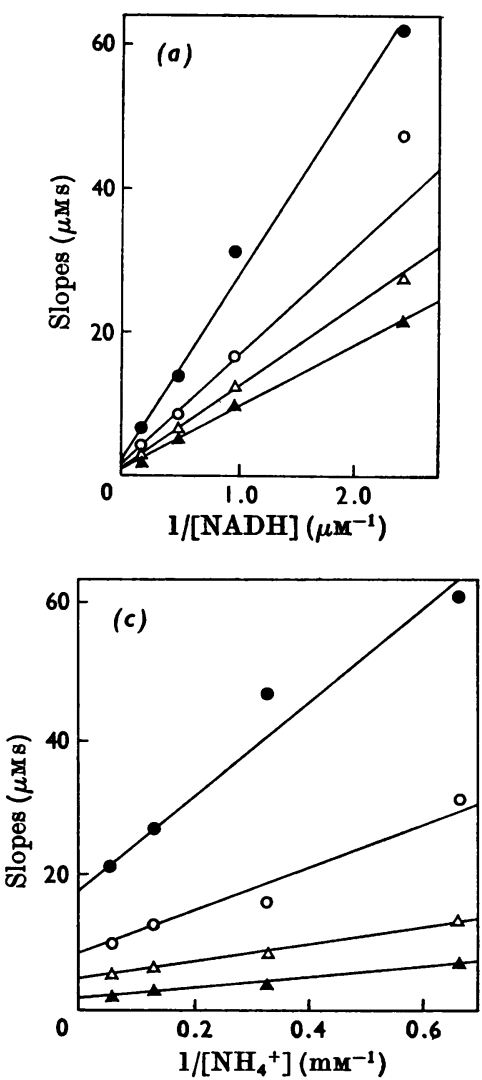
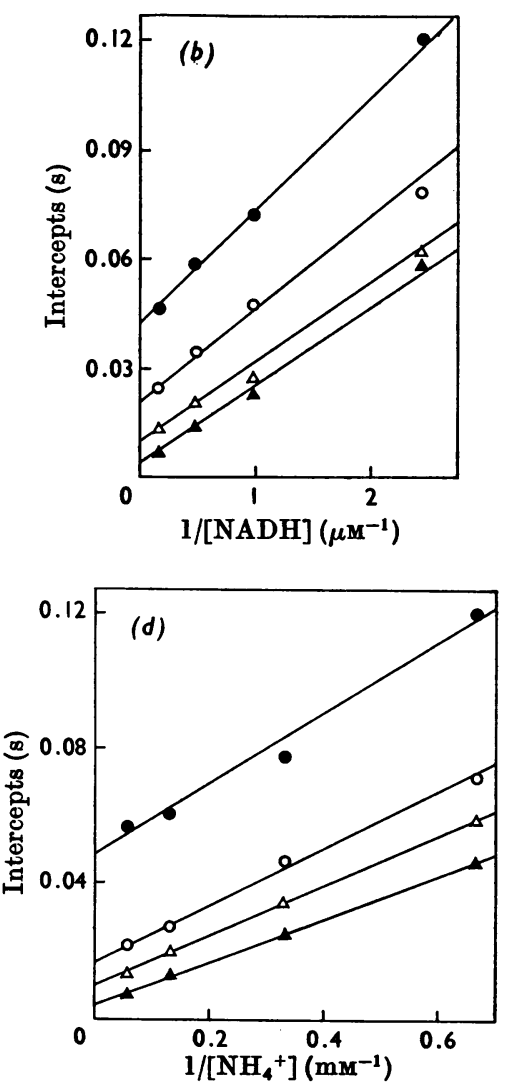

Fig. 2. Secondary plots of $(a)$ slopes and $(b)$ intercepts from Fig. 1 against the reciprocal of the NADH concentration for four fixed concentrations of $\mathrm{NH}_{4}{ }^{+}(\mathrm{mM}): 0,1.5 ; 0,3.0 ; \Delta, 7.5 ; \Delta, 18.0$. Secondary plots of $(c)$ slopes and $(d)$ intercepts from Fig. 1 against the reciprocal of the $\mathrm{NH}_{4}{ }^{+}$concentration for four fixed concentrations of NADH $(\mu \mathrm{M}): \bullet, 0.411 ; 0,1.03 ; \Delta, 2.05 ; \Delta, 6.15$. 

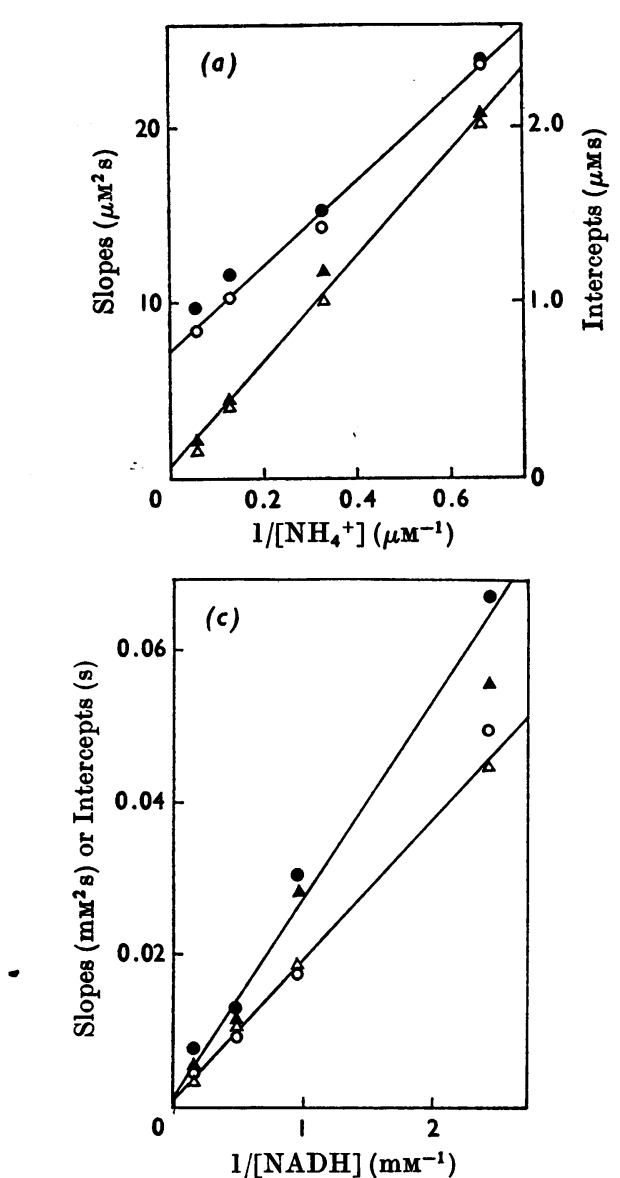
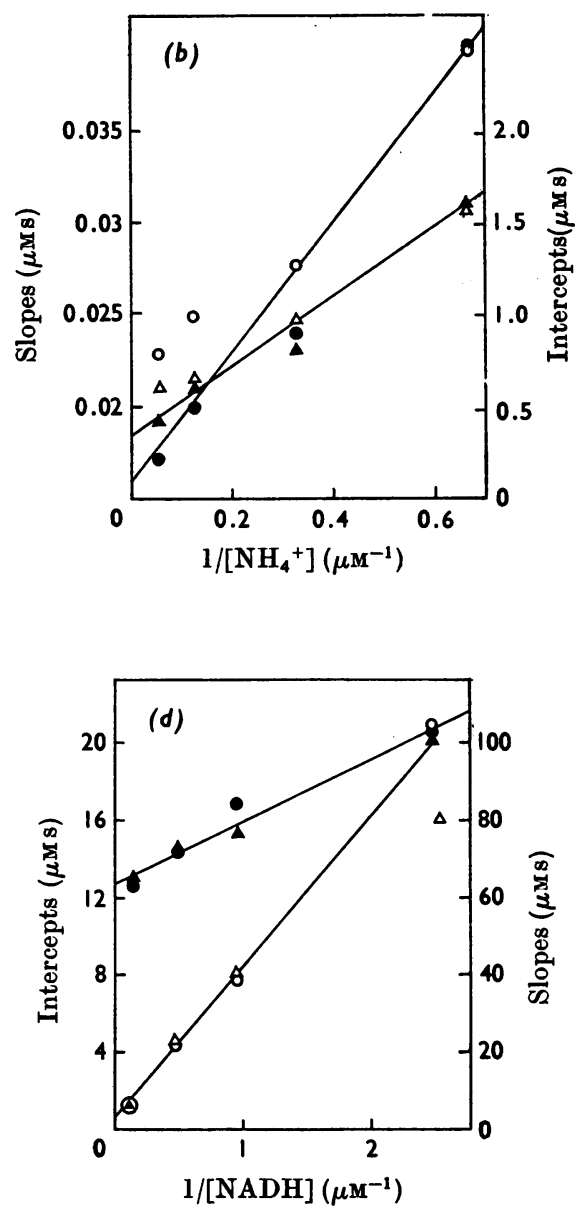

Fig. 3. Tertiary plots. $(a)$ The slopes $(O)$ from Fig. $2(a)$ and intercepts $(\Delta)$ from Fig. 2(b) are plotted against the reciprocal of the $\mathrm{NH}_{4}{ }^{+}$concentrations, and give, respectively, $\phi_{\mathrm{AC}}$ and $\phi_{0}$ as intercepts, and $\phi_{\mathrm{ABC}}$ and $\phi_{\mathrm{B}}$ as slopes. Also plotted are the slopes $(\bullet)$ and intercepts $(\Delta)$ of secondary plots (not shown) against the reciprocal of the 2-oxoglutarate concentration, derived from alternative primary plots of the data of Fig. 1 against the reciprocal of the NADH concentrations. (b) The slopes $(\Delta)$ from Fig. $2(b)$ and intercepts $(O)$ from Fig. 2(a) are plotted against the reciprocal of the $\mathrm{NH}_{4}{ }^{+}$concentration, and give, respectively, $\phi_{\mathrm{C}}$ and $\phi_{\mathrm{A}}$ as intercepts and $\phi_{\mathrm{BC}}$ and $\phi_{\mathrm{AB}}$ as slopes. As in $(a)$, slopes $(\Delta)$ and intercepts $(\bullet)$ from alternative secondary plots are also plotted. (c) The slopes (๑) from Fig. 2(c) and intercepts (O) from Fig. 2(d) are plotted against the reciprocal of the NADH concentration, and give, respectively, $\phi_{\mathrm{AB}}$ and $\phi_{0}$ as intercepts and $\phi_{\mathrm{ABC}}$ and $\phi_{\mathrm{C}}$ as slopes. Also plotted are slopes $(\Delta)$ and intercepts $(\Delta)$ from secondary plots with the 2-oxoglutarate concentration as variable, derived from primary plots with the $\mathrm{NH}_{4}{ }^{+}$concentration as variable. $(d)$ The slopes $(\bullet)$ from Fig. $2(d)$ and intercepts $(\Delta)$ from Fig. 2(c) are plotted against the reciprocal of the NADH concentration, and give respectively $\phi_{B}$ and $\phi_{A}$ as intercepts and $\phi_{B C}$ and $\phi_{A C}$ as slopes. As in $(c)$, slopes (O) and intercepts $(\Delta)$ from alternative secondary plots are also shown.

were given equal weight in estimating the initialrate parameters. The three sets of values so obtained are given in Table 1 , together with the mean values.

In a second experiment, five concentrations of each of the three substrates were used in the ranges 0.39-2.32 $\mu \mathrm{M}$-NADH, $\quad 0.15-2.0 \mathrm{~mm}$-2-oxoglutarate and 2.0-20.0 $\mathrm{mM}^{-} \mathrm{NH}_{4}{ }^{+}$, with enzyme concentrations of $0.057-0.23 \mu \mathrm{g} / \mathrm{ml}$. The mean values for the kinetic coefficients obtained by similar graphical analysis are also given in Table 1 (Expt. 2).

As is indicated in Table 1 , and is apparent in Figs. 3(c) and 3(d), only approximate estimates of $\phi_{0}, \phi_{\mathrm{A}}$ and $\phi_{\mathrm{AB}}$ could be obtained from these experiments. This is also true of $\phi_{\mathrm{BC}}$, although the 
Table 1. Kinetic coefficients for the reductive amination of 2-oxoglutarate in phosphate buffer, I0.25, at $25^{\circ} \mathrm{C}$

The kinetic coefficients are those in the initial-rate equation:

$$
\frac{e}{v_{0}}=\phi_{0}+\frac{\phi_{\mathrm{A}}}{[\mathrm{A}]}+\frac{\phi_{\mathrm{B}}}{[\mathrm{B}]}+\frac{\phi_{\mathrm{C}}}{[\mathrm{C}]}+\frac{\phi_{\mathrm{AB}}}{[\mathrm{A}][\mathrm{B}]}+\frac{\phi_{\mathrm{AC}}}{[\mathrm{A}][\mathrm{C}]}+\frac{\phi_{\mathrm{BC}}}{[\mathrm{B}][\mathrm{C}]}+\frac{\phi_{\mathrm{ABC}}}{[\mathrm{A}][\mathrm{B}][\mathrm{C}]}
$$

where $\mathrm{A}$ is 2-oxoglutarate, $\mathrm{B}$ is $\mathrm{NH}_{4}{ }^{+}$and $\mathrm{C}$ is $\mathrm{NADH}$ or NADPH. The kinetic coefficients were estimated as the slopes and intercepts of tertiary plots as described in the text. For Expt. 1 with NADH at pH 7.0, the tertiary plots are (i) Figs. 3(a) and 3(b); (ii) Figs. 3(c) and 3(d); (iii) not shown. For the experiments under other conditions, the ranges of values obtained from alternative tertiary plots are given.

\begin{tabular}{|c|c|c|c|c|c|c|c|c|}
\hline & $\begin{array}{c}10^{4} \phi_{0} \\
(\mathrm{~s})\end{array}$ & $\underset{(\mu \mathrm{M} s)}{\phi_{\mathbf{A}}}$ & $\begin{array}{c}\phi_{\mathrm{B}} \\
(\mu \mathrm{M} \mathrm{s})\end{array}$ & $\begin{array}{l}10^{3} \phi_{\mathrm{c}} \\
(\mu \mathrm{M} \mathrm{s})\end{array}$ & $\begin{array}{c}10^{-3} \phi_{\mathrm{AB}} \\
\left(\mu \mathrm{M}^{2} \mathrm{~g}\right)\end{array}$ & $\begin{array}{c}\phi_{\mathrm{AC}} \\
\left(\mu \mathbf{M}^{2} \mathrm{~B}\right)\end{array}$ & $\begin{array}{c}\phi_{\mathrm{BC}} \\
\left(\mu \mathrm{M}^{2} \mathrm{~B}\right)\end{array}$ & $\begin{array}{c}10^{-3} \phi_{\mathrm{ABC}} \\
\left(\mu \mathrm{M}^{3} \mathrm{~s}\right)\end{array}$ \\
\hline \multicolumn{9}{|l|}{ NADH, pH 7.0} \\
\hline Expt. (i) & 6.0 & 0.4 & 61 & 18.6 & 3.6 & 7.4 & 18.9 & 24.9 \\
\hline (ii) & 11.0 & 0.4 & 63 & 18.7 & 2.0 & 8.0 & 15.7 & 26.4 \\
\hline (iii) & 7.5 & 0 & 64 & 19.0 & 3.0 & 8.6 & 15.2 & 23.1 \\
\hline Average & 8.0 & 0.4 & 63 & 18.8 & 2.9 & 8.0 & 16.6 & 24.8 \\
\hline Expt. 2 Average & 10.0 & 0.6 & 70 & 22.0 & 4.0 & 8.5 & 20.0 & 27.0 \\
\hline NADH, pH 8.0 & $\begin{array}{r}7.0 \\
\pm 2.0\end{array}$ & $\begin{array}{c}0.5 \\
\pm 0.15\end{array}$ & $\begin{array}{l}15 \\
\pm 4\end{array}$ & $\begin{array}{l}24.0 \\
\pm 1.0\end{array}$ & $\begin{array}{r}1.6 \\
\pm 0.8\end{array}$ & $\begin{array}{r}23.5 \\
\pm 1.0\end{array}$ & $\begin{array}{r}29.0 \\
\pm 15.0\end{array}$ & $\begin{array}{r}23.0 \\
\pm 7.0\end{array}$ \\
\hline NADPH, pH 7.0 & $\begin{array}{r}16.0 \\
\pm 10.0\end{array}$ & $\begin{array}{r}0.15 \\
\pm 0.05\end{array}$ & $\begin{array}{l}61 \\
\pm 3\end{array}$ & $\begin{array}{r}27.0 \\
\pm 4.0\end{array}$ & $\begin{array}{r}0.5 \\
\pm 0.1\end{array}$ & $\begin{array}{r}10.0 \\
\pm 1.5\end{array}$ & $\begin{array}{r}22.0 \\
\pm 2.0\end{array}$ & $\begin{array}{r}16.0 \\
\pm 2.0\end{array}$ \\
\hline NADPH, pH 8.0 & $\begin{array}{r}17.0 \\
\pm 7.0\end{array}$ & $\begin{array}{r}1.65 \\
\pm 0.15\end{array}$ & $\begin{array}{r}\mathbf{2 4} \\
\pm 3\end{array}$ & $\begin{array}{r}28.0 \\
\pm 5.0\end{array}$ & $\begin{array}{r}4.4 \\
\pm 1.4\end{array}$ & $\begin{array}{r}24.0 \\
\pm 2.0\end{array}$ & $\begin{array}{l}17.5 \\
\pm 1.7\end{array}$ & $\begin{array}{r}17.3 \\
\pm 1.0\end{array}$ \\
\hline
\end{tabular}

estimates from different sets of plots are in good agreement with one another. The experimental uncertainties can also be seen from the secondary plots of Fig. 2. For example, $\phi_{\mathrm{AB}}$ is estimated from the change of intercepts with $\mathrm{NH}_{4}{ }^{+}$concentration in Fig. $2(a)$, and $\phi_{\mathrm{BC}}$ from the change of slopes with $\mathrm{NH}_{4}{ }^{+}$concentration in Fig. 2(b). The changes are small, and in a single experiment might beconsidered negligible. On the other hand, the variation of intercepts in Fig. 2(c) and of slopes in Fig. 2(a), which give $\phi_{\mathrm{AC}}$ and $\phi_{\mathrm{ABC}}$ respectively, are unequivocal. The limitations on the estimates of some of the kinetic coefficients at this $\mathrm{pH}$ value arise from their relative values and the upper limits imposed on the concentrations of NADH and 2-oxoglutarate by substrate inhibition, and on the $\mathrm{NH}_{4}{ }^{+}$concentration by the necessity of avoiding significant changes of ionic strength. There was no evidence of substrate activation or inhibition by $\mathrm{NH}_{4}^{+}$up to $51 \mathrm{~mm}$ (with 6.15 $\mu \mathrm{m}-\mathrm{NADH}$ and 3.0 mM-2-oxoglutarate). Substrate inhibition by NADH was observed with concentrations greater than $50 \mu \mathrm{M}$ (cf. Olson \& Anfinsen, 1953), and an increase of rate with time (Churchich \& Wold, 1963) under these conditions was also confirmed.

Kinetics with $N A D H$ at $\mathrm{pH}$ 8.0. In view of the complex pattern of 'coenzyme activation' observed in the oxidative deamination of glutamate (Engel \& Dalziel, 1969), it was decided to study the reverse reaction also with very wide ranges of concentration of each substrate and large, fixed concentrations of

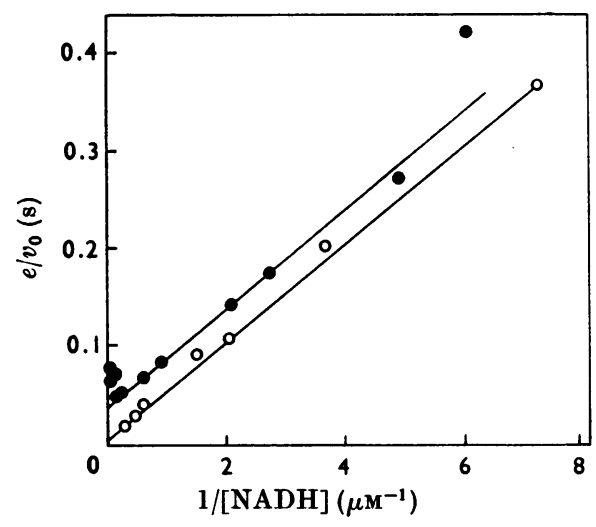

Fig. 4. Variation of the reciprocal of the specific initial rate with the reciprocal of the NADH concentration, with $0.80 \mathrm{~mm}$-2-oxoglutarate and $10 \mathrm{~mm}-\mathrm{NH}_{4}{ }^{+}$. Results with two concentration ranges of $\mathrm{NADH}$ are shown: $0,0.133-$ $3.3 \mu \mathrm{M} ; \bullet, 1.6-133 \mu \mathrm{M}$, in which plot the specific rates and NADH concentrations have been divided by 10. Phosphate buffer, pH8.0 and $I 0.25$, was used.

the other two. The results of initial-rate measurements with 15 concentrations of NADH from $0.133 \mu \mathrm{M}$ to $133 \mu \mathrm{M}$, and fixed concentrations of $0.80 \mathrm{~mm}$ 2-oxoglutarate and $10 \mathrm{~mm}-\mathrm{NH}_{4}{ }^{+}$, are shown in Fig. 4. The reciprocal plot is linear over the range 0.133-30 $\mu \mathrm{M}$-NADH, above which deviation towards 


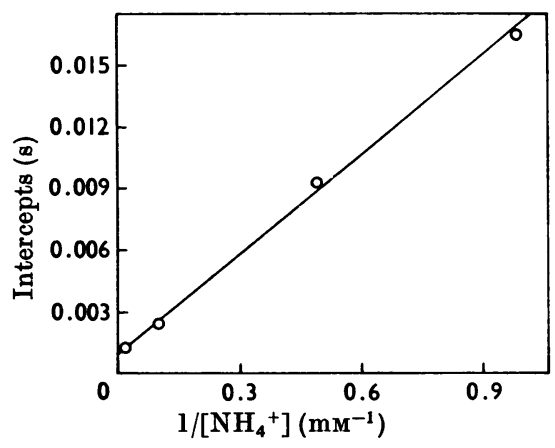

Fig. 5. Variation of the intercepts of secondary plots with the reciprocal of the concentration of $\mathrm{NH}_{4}{ }^{+}$. The intercepts plotted represent the reciprocals of the specific rate with infinitely large concentrations of NADH and 2-oxoglutarate. Values for $\phi_{0}$ and $\phi_{B}$ are obtained as the intercept and slope respectively of this tertiary plot. Phosphate buffer, pH 8.0 and I0.25, was used.

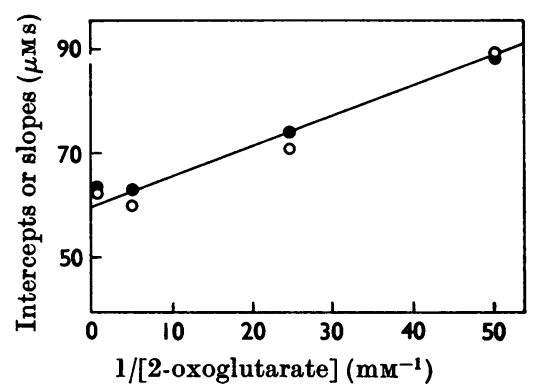

Fig. 6. Variation of the slopes (O) and intercepts (๑) of alternative secondary plots with the reciprocal of the concentration of 2-oxoglutarate, from which $\phi_{B}$ is obtained as the intercept and $\phi_{\mathrm{AB}}$ as the slope. The coenzyme was NADPH. Phosphate buffer, pH7.0, I0.25, was used.

lower activity occurs. Similarly, with $10.6 \mu \mathrm{M}$ $\mathrm{NADH}$ and $10 \mathrm{~mm}-\mathrm{NH}_{4}{ }^{+}$, a Lineweaver-Burk plot with respect to 2-oxoglutarate was linear in the range $0.01-1.0 \mathrm{~mm}$, and again showed substrate inhibition at higher concentrations $(2.0-20 \mathrm{~mm})$. Finally, with $10.6 \mu \mathrm{M}-\mathrm{NADH}$ and $0.80 \mathrm{~mm}$-2-oxoglutarate, the reciprocal rate was a linear function of the reciprocal of the concentration of $\mathrm{NH}_{4}{ }^{+}$from $0.1 \mathrm{~mm}$ to $35 \mathrm{~mm}$ (15 concentrations).

To estimate the initial-rate parameters, additional measurements were made with four concentrations of each substrate in all possible combinations, in the ranges $\quad 0.67-40 \mu \mathrm{M}-\mathrm{NADH}, \quad 0.05-3.0 \mathrm{~mm}$-2-oxoglutarate and 1.0-40 $\mathrm{mm}-\mathrm{NH}_{4}{ }^{+}$. Primary, secondary and tertiary plots were linear within the experimen-

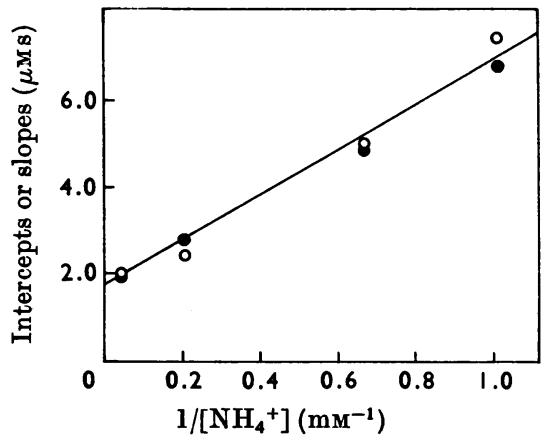

Fig. 7. Variation of the slopes (O) and intercepts $(\bullet)$ of alternative secondary plots with the reciprocal of the $\mathrm{NH}_{4}{ }^{+}$concentration, from which $\phi_{A}$ is obtained as intercept and $\phi_{\mathrm{AB}}$ as slope. The coenzyme was NADPH. Phosphate buffer, pH 8.0 and I0.25, was used.

tal error, and yielded approximate estimates of $\phi_{\mathrm{AB}}$, $\phi_{\mathrm{BC}}$ and $\phi_{\mathrm{ABC}}$, and fairly precise estimates of the other five kinetic coefficients. An example of a tertiary plot, from which one of the six estimates of $\phi_{0}$ and $\phi_{\mathrm{B}}$ were obtained, is shown in Fig. 5. Still further measurements were then made, on the basis of these results, with appropriate combinations of substrate concentrations, to refine the estimates of the kinetic coefficients by an iterative procedure. The final estimates, and the range of values from alternative plots, are given in Table 1.

Kinetics with $N A D P H$. Initial-rate measurements were made at $\mathrm{pH} 7.0$ with four concentrations of each substrate in the ranges 0.59-22.1 $\mu \mathrm{M}-\mathrm{NADH}$, $0.02-1.25 \mathrm{~mm}-2$-oxoglutarate and 0.5-25 $\mathrm{mM}^{-\mathrm{NH}_{4}}{ }^{+}$, with enzyme concentrations of $0.22-1.1 \mu \mathrm{g} / \mathrm{ml}$. Linear primary, secondary and tertiary plots were obtained, and the kinetic coefficients obtained from them are given in Table 1. Two tertiary plots, derived from alternative secondary plots, are shown in Fig. 6, and give $\phi_{\mathrm{B}}$ as intercept and $\phi_{\mathrm{AB}}$ as slope.

Similar measurements were made at $\mathrm{pH} 8.0$ with 0.7-17.4 $\mu \mathrm{M}-\mathrm{NADH}, \quad 0.05-1.25 \mathrm{mM}-2$-oxoglutarate,

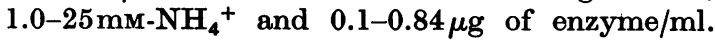
To obtain more precise estimates of $\phi_{\mathrm{BC}}$ and $\phi_{\mathrm{ABC}}$, additional measurements were then made with $0.05 \mu \mathrm{M}$ - and $0.10 \mu \mathrm{M}-\mathrm{NADH}, 0.10 \mathrm{mM}$ - and $0.20 \mathrm{mM}$ $\mathrm{NH}_{4}^{+}$, and 0.05-2.0 mM-2-oxoglutarate. Two tertiary plots, which give $\phi_{\mathrm{A}}$ as intercept and $\phi_{\mathrm{AB}}$ as slope, are shown in Fig. 7, and the kinetic coefficients obtained are given in Table 1.

\section{DISCUSSION}

Under the conditions of the above experiments, the initial rate of reductive amination of 2-oxoglutarate with either NADH or NADPH as coenzyme 
can be described by a linear reciprocal rate equation of the general form of eqn. (1) over wide ranges of all three substrate concentrations. Lineweaver-Burk plots were linear except with large conoentrations of NADH $(>30 \mu \mathrm{M})$ or 2-oxoglutarate (>2 mM), which caused deviations towards lower activity. There was no counterpart to the complex pattern of 'coenzyme aotivation' demonstrated for the reverse reaction and indicative of negative interactions (Dalziel \& Engel, 1968; Engel \& Dalziel, 1969).

As with glutamate oxidation, the enzyme utilizes both coenzymes with similar efficiency at both $\mathrm{pH}$ values studied. In general, the rate is slightly less with NADPH than with NADH; the maximum specific rate with NADH $\left(1 / \phi_{0}=1350 \mathrm{~s}^{-1}\right)$ is about twice that with NADPH, and is the same at both $\mathrm{pH} 7$ and $\mathrm{Ph}$. The Michaelis constants for the two coenzymes, i.e. the concentrations giving half the maximum rates with infinitely large concentrations of the other two substrates, $\phi_{\mathrm{c}} / \phi_{0}$, are also of the same order at both $\mathrm{pH}$ values, for example, $23 \mu \mathrm{M}$ for NADH at pH 7.0. However, at pH 7.0 with small concentrations of $\mathbf{2 . 0 x o g l u t a r a t e}$ and large concentrations of coenzyme, the rate with NADPH as coenzyme is greater than that with NADH, beoause $\phi_{A}$ and $\phi_{A B}$ are significantly smaller. The Michaelis constant for 2-oxoglutarate $\left(\phi_{\mathrm{A}} / \phi_{0}\right)$ is ten times as great at $\mathrm{pH} 8.0$ as at $\mathrm{pH} 7.0$ with $\mathrm{NADPH}$ as coenzyme, but does not change significantly with pH with NADH as coenzyme. With both coenzymea, the Michaelis constant for $\mathrm{NH}_{4}{ }^{+}\left(\phi_{\mathrm{B}} / \phi_{0}\right)$ is significantly less at $\mathrm{pH} 8.0$ than at $\mathrm{pH}$ 7.0.

At pH 7.0 with NADH as coenzyme, the Michaelis constants for 2.oxoglutarate and $\mathrm{NH}_{4}{ }^{+}$are about 0.5 and $0.08 \mathrm{~mm}$ respectively. These constants as defined above have no simple physiological significance. With $0.15 \mathrm{~mm}-2-0 x 0$ lutarate and $0.5 \mathrm{~mm}$ $\mathrm{NH}_{4}{ }^{+}$, the concentrations in the freeze-clamped liver of the well-fed rat (Williamson, Lund \& Krebs, 1967), and free NADH or NADPH concentrations of $1 \mu \mathrm{M}$ or more, the values for the initial-rate parameters show that the dominant terms in the rate equation are $\phi_{\mathrm{B}} /\left[\mathrm{NH}_{4}{ }^{+}\right]$and $\phi_{\mathrm{ABC}} /[\mathrm{NADH}][2$-oxoglutarate $]\left[\mathrm{NH}_{4}{ }^{+}\right]$.

Eqn. (1) is symmetrical with respect to the three substrates. Such a rate equation can be derived for a mechanism in which the three substrates combine randomly with the enzyme to give a quaternary complex (Mechanism 1), provided that all the complexes are assumed to be in rapid equilibrium with the substrates (Frieden, 1959). The rate equation is

$$
\frac{e}{v_{0}}=\frac{1}{k}+\frac{K_{\mathrm{BCA}}}{k[\mathrm{~A}]}+\frac{K_{\mathrm{ACB}}}{k[\mathrm{~B}]}+\frac{K_{\mathrm{ABC}}}{k[\mathrm{C}]}+\frac{\boldsymbol{K}_{\mathrm{ACB}} \boldsymbol{K}_{\mathrm{CA}}}{k[\mathrm{~A}][\mathrm{B}]}+\frac{\boldsymbol{K}_{\mathrm{ABC}} \boldsymbol{K}_{\mathrm{BA}}}{k[\mathrm{~A}][\mathrm{C}]}+\frac{K_{\mathrm{ABC}} \boldsymbol{K}_{\mathrm{AB}}}{k[\mathrm{~B}][\mathrm{C}]}+\frac{\boldsymbol{K}_{\mathrm{A}} \boldsymbol{K}_{\mathrm{AB}} \boldsymbol{K}_{\mathrm{ABC}}}{k[\mathrm{~A}][\mathrm{B}][\mathrm{C}]}
$$

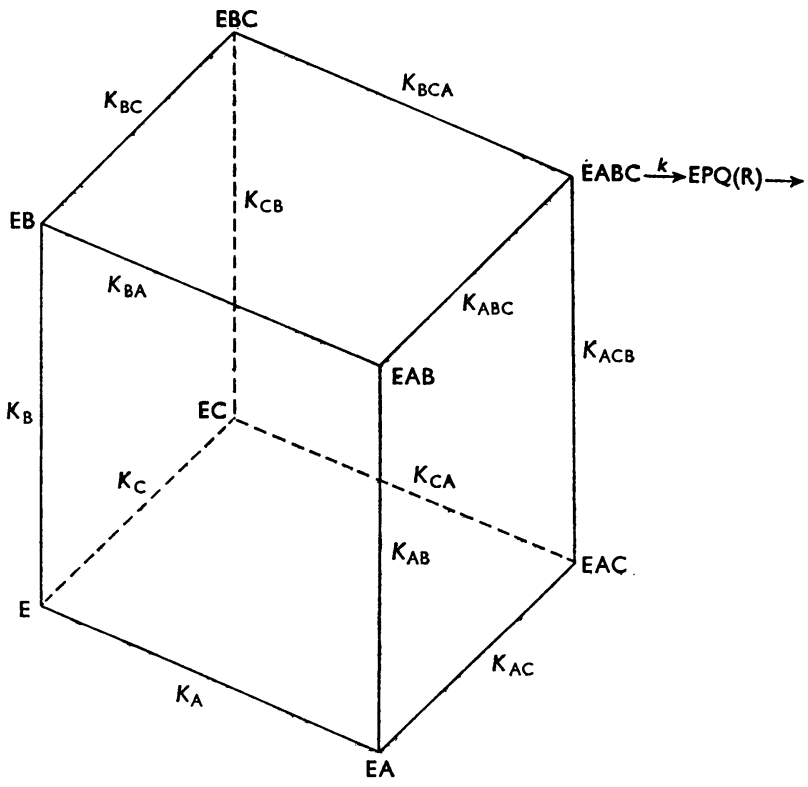

Mechanism 1 
where $K_{\mathrm{A}}, K_{\mathrm{BCA}}$ etc. are the dissociation constants of the complexes, and $k$ is the velocity constant for the rate-limiting step with saturating substrate concentrations.

Steady-state analyses of simpler mechanisms that do not involve alternative pathways also give rate equations of the same general form as eqn. (1) except that one or more of the eight terms is missing (Frieden, 1959). Thus for a strictly compulsoryorder mechanism in which $B$ is the second substrate to combine, the rate equation is
$\mathrm{pH} 7.0$ and pH8.0 (Engel \& Dalziel, 1969). This gives confidence in the reliability of the present measure. ments, especially as estimates of $\phi_{\mathrm{ABC}}$ rest heavily on rate measurements with small concentrations of all three substrates, made possible by the sensitivity of the fluorimetric method of analysis.

Similarly, the conclusive demonstration that the term $\phi_{\mathrm{AC}} /[\mathrm{A}][\mathrm{C}]$ is present in the rate equation (Figs. $3 a$ and $3 d$ ) eliminates the two possible compulsory-order mechanisms in which $\mathrm{NH}_{4}{ }^{+}$(B) is the second substrate. From the rate equation

$$
\frac{e}{v_{0}}=\phi_{0}+\frac{\phi_{\mathrm{A}}}{[\mathrm{A}]}+\frac{\phi_{\mathrm{B}}}{[\mathrm{B}]}+\frac{\phi_{\mathrm{C}}}{[\mathrm{C}]}+\frac{\phi_{\mathrm{AB}}}{[\mathrm{A}][\mathrm{B}]}+\frac{\phi_{\mathrm{BC}}}{[\mathrm{B}][\mathrm{C}]}+\frac{\phi_{\mathrm{ABC}}}{[\mathrm{A}][\mathrm{B}][\mathrm{C}]}
$$

where the kinetic coefficients are functions of the rate constants in the mechanism (Dalziel, 1969). The term $\phi_{\mathrm{AC}} /[\mathrm{A}][\mathrm{C}]$ in eqn. (1) is lacking, i.e. $\phi_{\mathrm{AC}}=0$. The second substrate to combine, $\mathrm{B}$, can therefore be identified, but the first and last substrate cannot be distinguished because eqn. (3) is symmetrical in $\mathbf{A}$ and $\mathbf{C}$.

Similarly, the steady-state rate equations for several possible enzyme-substitution mechanisms, in which intermediate group transfer to the enzyme, with product formation, occurs by reaction of only one or two of the three substrates, all lack the term $\phi_{\mathrm{ABC}} /[\mathrm{A}][\mathrm{B}][\mathrm{C}]$ of eqn. (1). The rate equations for these various mechanisms and their distinguishing features are discussed in detail elsewhere (Dalziel, 1969).

In the present experiments, the precision with which the eight kinetic coefficients in eqn. (1) could be estimated varied considerably. Accurate estimates of $\phi_{\mathrm{B}}, \phi_{\mathrm{C}}, \phi_{\mathrm{AC}}$ and $\phi_{\mathrm{ABC}}$ were readily obtained by graphical analysis of the results with both coenzymes and at both $\mathrm{pH}$ values. Estimates of the other four parameters were more uncertain because the occurrence of substrate inhibition prevented the use of sufficiently large concentrations of $\mathrm{NAD}(\mathrm{P}) \mathrm{H}$ or 2-oxoglutarate to make the terms containing them dominant under any conditions.

The clear-cut values obtained for $\phi_{\mathrm{ABC}}$ in all the experiments (cf. Figs. $3 a$ and $3 c$ ), conclusively eliminate all types of enzyme-substitution mechanism. Moreover, for the alternative quaternary complex mechanisms, whether random- or com. pulsory-order, this parameter should be related to the corresponding initial-rate parameter, $\phi_{\mathrm{PQ}}$, for the reverse reaction [in which only two substrates, glutamate and $N A D(P)$ are involved] and the equilibrium constant of the overall reaction, $K$. It has already been shown that this Haldane relation, $K=\phi_{\mathrm{ABC}} / \phi_{\mathrm{PQ}}$ (Dalziel, 1969), is satisfied very closely by results obtained with both coenzymes at for these mechanisms it can be shown that in addition to $\phi_{\mathrm{AC}}=0$, the relation $\phi_{\mathrm{ABC}} \phi_{\mathrm{B}} / \phi_{\mathrm{AB}} \phi_{\mathrm{B}} \mathrm{C}$ $=1$ must also be satisfied (Dalziel, 1969). Values for this ratio of kinetic coefficients, from Table 1 , are, at pH 7.0, 32 with NADH and 89 with NADPH, and at $\mathrm{pH} 8.0,7.4$ and 5.5 respectively. The mechanism proposed by Frieden (1959) from less detailed rate measurements in tris-acetate buffer, pH 8.0, in which the quaternary complex is formed by the successive combination of NADPH, $\mathrm{NH}_{4}{ }^{+}$and 2-oxoglutarate with the enzyme, is therefore inconsistent with the present results.

The two compulsory-order mechanisms with 2-oxoglutarate (A) as second substrate require $\phi_{\mathrm{BC}}=0$ and $\phi_{\mathrm{ABC}} \phi_{\mathrm{A}} / \phi_{\mathrm{AB}} \phi_{\mathrm{AC}}=1$. The values for the latter ratio, with both NADH and NADPH, are 0.5 at $\mathrm{pH} 7.0$ and 0.3 at $\mathrm{pH}$ 8.0. The deviation from unity is not significant at pH 7.0, especially as $\phi_{A}$ can only be estimated approximately (Fig. 3d), but at $\mathrm{pH} 8.0$ the deviation appears to be significant (Fig. 7). Moreover, although $\phi_{\mathrm{BC}}$ is small and a single estimate could not be considered significant, the fact that real, positive values for this kinetic coefficient were obtained in separate experiments with two coenzymes and at two $\mathrm{pH}$ values, and that for each experiment six estimates from alternative plots were in reasonably good agreement with one another, leads to the conclusion that these strictly compulsory-order mechanisms are not satisfactory.

The same considerations apply to the remaining two compulsory-order mechanisms with $\mathrm{NAD}(\mathrm{P}) \mathrm{H}$ as second substrate, which require $\phi_{\mathrm{AB}}=0$ (cf. Fig. 7) and $\phi_{\mathrm{ABC}} \phi_{\mathrm{C}} / \phi_{\mathrm{AC}} \phi_{\mathrm{BC}}=1$. With NADH as coenzyme, the latter ratio is 3.5 at $\mathrm{pH} 7.0$ and 2.0 at pH 8.0. These mechanisms, and two of those previously discussed in which the coenzyme is the last substrate to add, might be considered unlikely because it is known that the coenzyme will combine with the enzyme in the absence of the other two substrates. It may be added that for these com- 
pulsory-order mechanisms in which the coenzyme is not the leading substrate, the values of some of the initial-rate parameters and of ratios between them should be the same whether NADH or NADPH is used (Dalziel, 1969). These requirements also are not met, as shown by Engel (1968).

Partially random-order mechanisms, in which one substrate must add first, second or third and the other two combine randomly, yield complex, nonlinear reciprocal rate equations by steady-state analysis, and unlike a fully random-order mechanism cannot be treated by the equilibrium assumption (Dalziel, 1969). Such a mechanism, in which the coenzyme necessarily combines first with the enzyme and the other two substrates then combine randomly, seems a likely one for glutamate dehydrogenase. It can be shown that if certain inequalities between rate constants are assumed, the steadystate rate equation for this mechanism simplifies to the linear reciprocal form of eqn. (1), but lacks the terms in $\phi_{\mathrm{AB}}$ and $\phi_{\mathrm{AC}}$ (Dalziel, 1969). This mechanism is therefore also inconsistent with the present data.

It is concluded that the kinetic results cannot be accounted for by any enzyme-substitution or strictly compulsory-order mechanism, and that the mechanism is of the random-order type, with restrictions on the values of the rate constants such that the rate equation takes the simple linear reciprocal form of eqn. (1) over wide ranges of substrate concentrations. One such set of restric- tions is the rapid-equilibrium case which yields eqn. (2), and is well established for several twosubstrate reactions (cf. Morrison \& Cleland, 1966). For this case, the dissociation constants of all the enzyme-substrate complexes in Mechanism 1 may be calculated from ratios of the kinetic coefficients (Dalziel, 1969). The values for pH 7.0 with NADH as coenzyme are given in Table 2. The dissociation constants of the three binary and three ternary complexes could be estimated by direct binding studies, and comparison with the calculated values in Table 2, and corresponding values from the kinetic coefficients with NADPH as coenzyme (Table 1) would provide a test of the validity of the rapid-equilibrium random-order mechanism. At present, the only direct estimates available are for the enzyme-NADH compound, and the values, in various buffers and at various temperatures, cover a wide range. In $0.05 \mathrm{M}$-sodium phosphate buffer, pH 7.5, at $15^{\circ} \mathrm{C}$, Churchich (1967) obtained a value of $3.2 \mu \mathrm{M}$ by measurements of fluorescence polarization, and in $0.11 \mathrm{~m}$-sodium phosphate buffer at pH 7.0 and $23.5^{\circ} \mathrm{C}$, fluorescence-enhancement measurement gave $15 \mu \mathrm{M}$ (F. M. Dickinson, unpublished work), compared with the calculated value of $8.6 \mu \mathrm{M}$ (Table 2). Direct measurements of the dissociation constants of the binary complexes of enzyme with 2-oxoglutarate and $\mathrm{NH}_{4}{ }^{+}$, and of the effects of each of these substrates on the binding of NADH, would provide more critical tests of the mechanism. The results in Table 2 indicate that the

Table 2. Dissociation constants for enzyme-substrate complexes of glutamate dehydrogenase in phosphate buffer, $\mathrm{pH} 7.0$ and $I 0.25$, at $25^{\circ} \mathrm{C}$, calculated from kinetic data with $N A D H$ as coenzyme, on the basis of a rapid-equilibrium random-order mechanism

The dissociation constants are calculated as ratios of the kinetic coefficients in Table 1 , by relations derived from eqn. (2) for Mechanism 1 in the text (Dalziel, 1969). A is 2-oxoglutarate, $\mathrm{Bis}_{\mathrm{NH}_{4}}^{+}$and Cis NADH. Values in parentheses are from kinetic coefficients with NADPH as coenzyme.

Dissociating substrate

\begin{tabular}{llll}
\cline { 2 - 2 } & $\begin{array}{c}\text { NADH } \\
(\mu \mathrm{M})\end{array}$ & $\begin{array}{c}2 \text {-Oxoglutarate } \\
(\mu \mathrm{M})\end{array}$ & $\begin{array}{l}\mathrm{NH}_{4}^{+} \\
(\mathrm{mM})\end{array}$ \\
EABC & $\frac{\phi_{\mathrm{C}}}{\phi_{0}}=24$ & $\frac{\phi_{\mathrm{A}}}{\phi_{0}}=560$ & $\frac{\phi_{\mathrm{B}}}{\phi_{0}}=79$ \\
EAB & - & $\frac{\phi_{\mathrm{AC}}}{\phi_{0}}=430(370)$ & $\frac{\phi_{\mathrm{BC}}}{\phi_{\mathrm{C}}}=0.88(0.81)$ \\
EBC & $\frac{\phi_{\mathrm{AC}}}{\phi_{\mathrm{A}}}=18$ & - & $\frac{\phi_{\mathrm{AB}}}{\phi_{\mathrm{A}}}=6.4$ \\
EAC & $\frac{\phi_{\mathrm{BC}}}{\phi_{\mathrm{B}}}=0.26$ & $\frac{\phi_{\mathrm{AB}}}{\phi_{\mathrm{B}}}=46$ & - \\
EC, EA, EB & $\frac{\phi_{\mathrm{ABC}}}{\phi_{\mathrm{AB}}}=8.6$ & $\frac{\phi_{\mathrm{ABC}}}{\phi_{\mathrm{BC}}}=1490(730)$ & $\frac{\phi_{\mathrm{ABC}}}{\phi_{\mathrm{AC}}}=3.1(1.6)$
\end{tabular}


dissociation constant for NADH from the ternary complex with 2-oxoglutarate (EAC) is smaller by a factor of 30 than that for the binary E.NADH complex, and should be susceptible to direct measurement by the effect of 2-oxoglutarate on the fluorescence of $\mathrm{E} \cdot \mathrm{NADH}$, in the absence of $\mathrm{NH}_{4}{ }^{+}$. On the other hand, binding of $\mathrm{NH}_{4}+$ to the enzyme appears to halve its affinity for NADH (Table 2). The binding of 2-oxoglutarate to glutamate dehydrogenase in the absence of $\mathrm{NADH}$ and $\mathrm{NH}_{4}{ }^{+}$is also indicated by the finding that this substrate, and also NAD, glutamate and inhibitors that compete with the substrates, protect the enzyme from activation by phenylmercuric acetate (Mildvan \& Greville, 1962; A. S. Mildvan, personal communication). Further, Hochreiter \& Schellenberg (1969) have shown that the enzyme catalyses the formation of glutamate from 2-oxoglutarate and $\mathrm{NH}_{4}{ }^{+}$with dithionite as reductant, in the absence of coenzyme.

The dissociation constants of the ternary complex of enzyme, 2-oxoglutarate and $\mathrm{NH}_{4}{ }^{+}$, and of the binary complexes with these substrates, should be independent of the coenzyme, and therefore values for these constants calculated from kinetic coefficients obtained with NADH and NADPH as coenzyme should be the same. As is shown in Table 2, at pH 7.0 this is so for the ternary complex EAB, but there are discrepancies for the binary complexes EA and EB. Because of the experimental error in estimates of $\phi_{\mathrm{BC}}$, the discrepanoy is not significant for $K_{\mathrm{A}}$, but appears to be outside the experimental error for $K_{\mathrm{B}}$. By using the kinetic coefficients for pH 8.0 (Table 1), however, there is good agreement between the two estimates of these dissociation constants: $K_{\mathrm{A}}$ is $0.79 \mathrm{~mm}$ with $\mathrm{NADH}$ and $0.99 \mathrm{~mm}$ with NADPH, and the values for $K_{B}$ are $0.98 \mathrm{~mm}$ and $0.72 \mathrm{~mm}$ respectively.

Other special cases of a random-order mechanism, or partially random-order mechanism, may also account for the results. In addition to the determination of dissociation constants by direct binding studies, the estimation of rate constants for the binding and dissociation of the coenzyme by rapidreaction technique and studies of isotope exchange at equilibrium (cf. Morrison \& Cleland, 1966) are needed for further evaluation of the mechanism.

Although the values of initial-rate parameters would be expected to change with the nature of the buffer used, and with the ionic strength, it seems unlikely that a random-order mechanism in phosphate buffer is replaced by the strictly compulsory sequences $\mathrm{NADH}-\mathrm{NH}_{4}{ }^{+}$-2-oxoglutarate in tris-acetate buffer (Frieden, 1959) and NADH2-oxoglutarate- $\mathrm{NH}_{4}{ }^{+}$in arsenate buffer (Fahien \& Strmecki, 1969). It is necessary to point out briefly that the earlier conclusions were not justified by the limited experimental data obtained, which could be more simply accounted for by a random- order mechanism. The evidence for a compulsoryorder mechanism with $\mathrm{NH}_{4}{ }^{+}$as second substrate was the finding that with a large, fixed concentration of $\mathrm{NH}_{4}^{+}$, plots of the reciprocal rate against the reciprocal of the NADPH concentration for three different concentrations of 2-oxoglutarate were 'almost parallel' (Frieden, 1959). If the fixed concentration of $\mathrm{NH}_{4}{ }^{+}$were truly 'saturating', and such plots were really parallel, this would indicate that $\phi_{\mathrm{AC}}=0$, and the conclusion would be valid. In fact, the slopes of the plots varied by a factor of 1.8 over the range of concentration of 2-oxoglutarate used, and in the absence of more detailed measurements with several concentrations of $\mathrm{NH}_{4}{ }^{+}$, no conclusions can be drawn about the values of individual initial-rate parameters. It was also found that Lineweaver-Burk plots with NADPH as variable for three concentrations of $\mathrm{NH}_{4}{ }^{+}$, with a single large concentration of 2-oxoglutarate, intersected on the abscissa. Similarly, with a single, large concentration of NADPH and three concentrations of $\mathrm{NH}_{4}{ }^{+}$, Lineweaver-Burk plots with respect to 2-oxoglutarate intersected on the abscissa. If the mechanism is a random one, these findings would merely mean that the binding of $\mathrm{NH}_{4}{ }^{+}$to the ternary complex of the enzyme and the other two substrates does not significantly affect their dissociation constants. On the assumption of a compulsory-order mechanism, however, these findings mean that the velocity constants for the dissociation of $\mathrm{NH}_{4}{ }^{+}$from E.NADPH. $\mathrm{NH}_{4}{ }^{+}$and of NADPH from E.NADPH are fortuitously equal to one another and to the maximum specific rate of the reductive-amination reaction (Frieden, 1959). Moreover, the observed maximum specific rate of reductive amination is much greater than the rate of dissociation of E.NAD calculated on the assumption of a simple compulsory-order mechanism, in which this dissociation would be thelast step. This anomaly has been taken to indicate the existence of more than one form of E.NAD complex (Cleland, 1963). If the mechanism is a random one, however, as the present results indicate, no such anomaly exists, since the rate of dissociation of this complex cannot be calculated from the data.

We are indebted to the Medical Research Council for financial support.

\section{REFERENCES}

Churchich, J. E. (1967). Biochim. biophys. Acta, 147, 32. Churchich, J. E. \& Wold, F. (1963). Biochemistry, Easton, 2, 781 .

Cleland, W. W. (1963). Biochim. biophys. Acta, 67, 104. Corman, L., Prescott, L. M. \& Kaplan, N. O. (1967). J.biol. Chem. 242, 1383.

Dalziel, K. (1962). Biochem. J. 84, 240.

Dalziel, K. (1969). Biochem.J. 114, 547. 
Dalziel, K. \& Engel, P. C. (1968). FE BS Lett. 1, 349. Engel, P. C. (1968). D.Phil. Thesis: University of Oxford. Engel, P. C. \& Dalziel, K. (1969). Biochem. J. 115, 621.'

Fahien, L. A. \& Strmecki, M. (1969). Archs Biochem. Biophys. 130, 468.

Frieden, C. (1959). J.biol. Chem. 234, 2891.

Hochreiter, M. C. \& Schellenberg, K. A. (1969). J. Am. chem. Soc. 91, 6530.

Horecker, B. L. \& Kornberg, A. (1948). Biochem. J. 175 385.
Londesborough, J. C. \& Dalziel, K. (1968). Biochem. J. $110,217$.

Mildvan, A. S. \& Greville, G. D. (1962). Biochem. J. 84, $21 \mathrm{P}$.

Morrison, J. F. \& Cleland, W. W. (1966). J. biol. Chem. 241, 673.

Olson, J. A. \& Anfinsen, C. B. (1953). J. biol. Chem. 202, 841.

Williamson, D. H., Lund, P. \& Krebs, H. A. (1967). Biochem. J. 103, 514. 